\title{
ON K-TRANSFORM
}

\author{
C. NASIM \\ Department of Mathematics \\ The University of Calgary \\ Calgary, Alberta \\ Canada
}

(Recelved March 12, 1980)

ABSTRACT.

Using a combination of infintte order linear differential operators and Integral operators, the inversion of $K$-transform 18 established. Inversion procedures for Laplace transform and Potential transform are derived as special cases.

KEY WORDS AND PHRASES. K-transform, Laplace transform, differentlal operators. 1980 MATHEMATICS SUBJECT CLASSIFICATION CODES. 0029

1. INTRODUCTION.

In this paper, we discuss the inversion of the K-transform, in Hilbert space $L^{2}(0, \infty)$. The method involves only the real analysis and employs differential operators of infinite order, cf [4] and [6, Chapter vil] . An algorithm for 
inverting the bilateral Laplace transform is established as a special case. Also some examples are given to illustrate the procedure.

LEMMA 1. [5, p.94] Let $f(x) \in L^{2}(0, \infty)$ and

$$
F(s)=\int_{0}^{\infty} f(x) x^{s-1} d x, \quad s=\frac{1}{2}+i t, \quad-\infty<t<\infty,
$$

then

$$
f(x)=\frac{1}{2 \pi i} \lim _{t \rightarrow \infty} \int_{\frac{1 / 2}{2}-i t}^{\frac{1}{2}+i t} F(s) x^{-s} d s .
$$

$F$ is called the Mellin transform of $f$.

LEMMA 2. [5, p.95] If $f$ and $g \in L^{2}(0, \infty)$ and have Mellin transforms $F$ and $G$ respectively, then

$$
\int_{0}^{\infty} \frac{1}{x} f\left(\frac{1}{x}\right) g(x t) x^{s-1} d x=\frac{1}{2 \pi i} \int_{\frac{1}{2}-i_{\infty}^{\infty}}^{1 / 2+i \infty} F(s) G(s) t^{-s} d s .
$$

Next some operational considerations.

If $\theta=-x \frac{d}{d x}$, then it is an easy matter to see that

$$
\theta^{n}\left[x^{-s}\right]=s^{n} x^{-s}, \quad s=\sigma+i t, \quad-\infty<t<\infty .
$$

Therefore

$$
p_{n}(\theta)\left[x^{-s}\right]=p_{n}(s) x^{-s},
$$

where $p_{n}(\theta)$ is a polynomial of degree $n$ in $\theta$; consequently

$$
\begin{aligned}
p(\theta)\left[x^{-s}\right] & =\lim _{n \rightarrow \infty} p_{n}(\theta)\left[x^{-s}\right] \\
& =p(s) x^{-s} .
\end{aligned}
$$


Also,

$$
\begin{aligned}
n^{\theta}\left[x^{-s}\right] & =e^{\theta} \ln n\left[x^{-s}\right] \\
& =\lim _{N \rightarrow \infty} \sum_{k=0}^{N} \frac{(\theta \ln n)^{k}}{k !}\left[x^{-s}\right] \\
& =\lim _{N \rightarrow \infty} \sum_{k=0}^{N} \frac{(s \ln n)^{k}}{k !} x^{-s}=n^{s} x^{-s} .
\end{aligned}
$$

With this understanding, we interpret the operator $\frac{1}{\Gamma(1-\theta)}$, a linear differential operator of infinite order, [6, p.234], such that

$$
\begin{aligned}
\frac{1}{\Gamma(1-\theta)}\left[x^{-s}\right] & =\lim _{n \rightarrow \infty} n^{\theta} \prod_{k=1}^{n}\left(1-\frac{\theta}{k}\right)\left[x^{-s}\right] \\
& =\frac{1}{\Gamma(1-s)} x^{-s} .
\end{aligned}
$$

Similarly

$$
\frac{1}{\Gamma(\alpha-\beta \theta)}\left[x^{-s}\right]=\frac{1}{\Gamma(\alpha-\beta s)} x^{-s} .
$$

Next we define the operator $\Gamma(\alpha-\beta \theta)$ as having the property that

$$
\Gamma(\alpha-\beta \theta)\left[x^{-s}\right]=\Gamma(\alpha-\beta s) x^{-s}
$$

This is not a differential operator in the above sense, but behaves in the manner of (1.2) for all $s=\sigma+i t,-\infty<t<\infty, R(s)<\frac{\alpha}{\beta}$.

\section{THE MAIN RESULT}

THEOREM 1. Let the functions $f$ and $\phi \in L^{2}(0, \infty)$. Define

$$
f(x)=\left(\frac{2}{\pi}\right)^{\frac{1}{2}} \int_{0}^{\infty}(x t)^{\alpha} K_{\mu}(x t) \phi(t) d t,
$$

where $|\mu| \leq \frac{1}{2}, \alpha>0$ and $K_{\mu}$ being the usual modified Bessel function of order $\mu$. 
If

$$
R(x)=\left(\frac{2}{\pi}\right)^{\frac{3}{2}} \int_{0}^{\infty}(x t)^{\beta} J_{v}(x t) f(t) d t,
$$

$|\nu| \leq \frac{1}{2}, \quad B>0$ then

$$
\frac{\pi 2^{-(\alpha+\beta-1)} \Gamma\left[\frac{1}{2}(\nu-\beta+2-\theta)\right]}{\Gamma\left[\frac{1}{2}(\mu+\alpha+1-\theta)\right] \Gamma\left[\frac{1}{2}(\nu+\beta+\theta)\right] \Gamma\left[\frac{1}{2}(\alpha-\mu+1-\theta)\right]}[R(x)]=\phi(x), \text { a.a. } x>0 .
$$

PROOF. Note that the integral defining the function $f$ is absolutely convergent, since $\phi \in L^{2}(0, \infty)$ and $t^{\alpha} K_{\mu}(t) \in L^{2}(0, \infty)$ due to the hypotheses.

Now,

$$
\begin{aligned}
R(x) & =\left(\frac{2}{\pi}\right)^{\frac{1}{2}} \int_{0}^{\infty}(x t)^{\beta} J_{\nu}(x t) f(t) d t \\
& =\frac{2}{\pi} \int_{0}^{\infty}(x t)^{\beta} J_{\nu}(x t) d t \int_{0}^{\infty}(u t)^{\alpha} K_{\mu}(u t) \phi(u) d u \\
& =\frac{2}{\pi} x^{\beta} \int_{0}^{\infty} u^{\alpha} \phi(u) d u \int_{0}^{\infty} t^{\alpha+\beta} J_{\nu}(x t) K_{\mu}(u t) d t .
\end{aligned}
$$

The change of order of integration is justified by absolute convergence

$$
\int_{0}^{\infty} \int_{0}^{\infty}\left|t^{\alpha+\beta} u^{\alpha} J_{v}(x t) K_{\mu}(u t) \phi(u)\right| d u d t<\infty
$$

using the asymptotic expansions of the Bessel functions $J$ and $K$. The $t$-integral can be evaluated, [I(II), p.137] so that (2.1) above gives,

$$
R(x)=\frac{2^{\alpha+\beta}}{\pi} \frac{\Gamma(a) \Gamma(b)}{\Gamma(v+1)} \int_{0}^{\infty} x^{\beta+v} u^{-(v+\beta+1)} F_{1}\left(a, b ; v+1 ; \frac{-x^{2}}{u^{2}}\right) \phi(u) d u,
$$

where $R(\nu+1+\alpha+\beta)>|R(\mu)|$ and $a=\frac{1}{2}(\nu+\mu+1+\alpha+\beta), b=\frac{1}{2}(\nu-\mu+1+\alpha+\beta),{ }_{2} F_{1}$ being the Hypergeometric function. 
Let,

where

$$
R(x)=\int_{0}^{\infty} \frac{1}{u} \phi\left(\frac{1}{u}\right) k(x u) d u,
$$

$$
k(x)=\frac{2^{\alpha+\beta}}{\pi} \frac{\Gamma(a) \Gamma(b)}{\Gamma(v+1)} x_{2}^{\alpha+\beta} F_{1}\left(a, b ; v+1 ;-x^{2}\right) \text {. }
$$

Now $k \in L^{2}(0, \infty)$ since $\beta+\nu>-\frac{1}{2}$ and $|\mu|<\alpha+\frac{1}{2}$, and further $\frac{1}{x} \phi\left(\frac{1}{x}\right) \in L^{2}(0, \infty)$, since $\phi$ does, hence by Lemma 2 , we have

$$
R(x)=\frac{1}{2 \pi i} \int_{\frac{1}{2}-i \infty}^{\frac{1}{2}+i \infty} \Phi(s) K(s) x^{-s} d s,
$$

where $\Phi$ and $K$ are the Mellin transforms of $\phi$ and $k$ respectively and $[1(I), p .336]$,

$$
K(s)=\frac{2^{\alpha+\beta-1}}{\pi} \frac{\Gamma\left[\frac{1}{2}(\mu+\alpha+1-s)\right] \Gamma\left[\frac{1}{2}(\nu+\beta+s)\right] \Gamma\left[\frac{1}{2}(\alpha-\mu+1-s)\right]}{\Gamma\left[\frac{1}{2}(\nu-\beta+2-s)\right]}
$$

Next, we apply the operator $\frac{1}{K(\theta)}$, where $\theta=-x \frac{d}{d x}$ to the function $R(x)$, to obtain

$$
\begin{aligned}
\frac{1}{K(\theta)}[R(x)] & =\frac{1}{K(\theta)}\left[\frac{1}{2 \pi i} \int_{\frac{1}{2}-i \infty}^{\frac{1}{2}+i \infty} \Phi(s) K(s) x^{-s} d s\right] \\
& =\frac{1}{2 \pi i} \int_{\frac{1}{2}-i \infty}^{\frac{1}{2}+i \infty} \Phi(s) K(s) d s \frac{1}{K(\theta)}\left[x^{-s}\right] .
\end{aligned}
$$

Using the results (1.1) and (1.2), we see that $\frac{1}{K(\theta)}\left[x^{-s}\right]=\frac{1}{K(s)} x^{-8}$. Thus,

$$
\begin{aligned}
\frac{1}{K(\theta)}[R(x)] & =\frac{1}{2 \pi i} \int_{\frac{1}{2}-i \infty}^{\frac{1}{2}+i \infty} \Phi(s) K(s) \frac{x^{-s}}{K(s)} d s \\
& =\frac{1}{2 \pi i} \int_{\frac{1}{2}-i \infty}^{\frac{1}{2}+i \infty} \Phi(s) x^{-s} d s \\
& =\Phi(x), \text { a.a. } x>0,
\end{aligned}
$$

as required. The bringing of the operator $\frac{1}{K(\theta)}$ inside the integral, amounts to 
nothing more than differentiating inside the integral sign.

EXAMPLE 1. Let $\phi(x)=J_{\eta}(x)$. Then

$$
\begin{aligned}
f(x)= & \sqrt{\frac{2}{\pi}} \int_{0}^{\infty}(x t)^{\alpha} J_{n}(t) K_{\mu}(x t) d t \\
= & \frac{2^{\alpha-\frac{1}{2}}}{\pi^{\frac{1}{2}}} x^{-(n+1) \frac{\Gamma\left[\frac{1}{2}(n+\mu+\alpha+1)\right] \Gamma\left[\frac{1}{2}(n-\mu+\alpha+1)\right]}{\Gamma(n+1)}} \\
& \quad{ }_{2} F_{1}\left(\frac{1}{2}(n+\mu+\alpha+1), \frac{1}{2}(n-\mu+\alpha+1) ; n+1 ;-\frac{1}{x^{2}}\right)
\end{aligned}
$$

[1(II), p.137]. And

$$
R(x)=\frac{2^{\alpha}}{\pi} \frac{\Gamma(c) \Gamma(d)}{\Gamma(n+1)} x^{\beta} \int_{0}^{\infty} t^{\beta-n-1}{ }_{2} F_{1}\left(c, d ; n+1 ;-\frac{1}{t^{2}}\right) J_{v}(x t) d t
$$

where $c=\frac{1}{2}(n+\mu+\alpha+1), d=\frac{1}{2}(n-\mu+\alpha+1)$.

$$
=\frac{2^{\alpha+\beta-n-1}}{\pi} x^{n} G_{24}^{22}\left(\frac{\frac{1}{4}}{4} x^{2} \mid \begin{array}{l}
1-c, 1-\alpha \\
\frac{1}{2}(\beta-n+\nu), 0,-n, \frac{1}{2}(\beta-\eta-\nu)
\end{array}\right),
$$

[1(II), p.82], where $G$ is Meijer's $G$ Function.

Now

$$
\begin{aligned}
\frac{1}{K(\theta)} R(x)= & \frac{2^{-\eta} \Gamma\left[\frac{1}{2}(\nu-\beta+2-\theta)\right]}{\Gamma\left[\frac{1}{2}(\mu+\alpha+1-\theta)\right] \Gamma\left[\frac{1}{2}(\nu+\beta+\theta)\right] \Gamma\left[\frac{1}{2}(\alpha-\mu+1-\theta)\right]} \\
& \quad\left[x^{n} G_{24}^{22}\left(\frac{1}{4} x^{2} \mid \begin{array}{l}
1-c, 1-d \\
\frac{1}{2}(\beta-\eta+\nu), 0,-n, \frac{1}{2}(\beta-\eta-\nu)
\end{array}\right)\right] \\
= & 2^{-n} x^{n} G_{02}^{10}\left(\frac{1}{4} x^{2} \mid 0,-\eta\right) \\
& =J_{n}(x), \text { as predicted. }
\end{aligned}
$$


The operator $\frac{1}{K(\theta)}$ applied on $x^{n} G_{24}^{22}(\cdot)$, is evaluated by writing $G_{24}^{22}(\cdot)$ in terms of the complex integral and then the differential operator being applied inside the integral to give $x^{n} G_{02}^{10}(\cdot)$.

\section{SPECIAL CASES.}

(i) Let $\alpha+\beta-1=\mu+\nu$. The inversion of the main theorem $b_{2}$

$$
\frac{\pi}{2^{\mu+\nu}} \frac{1}{\Gamma\left[\frac{1}{2}(\mu+\alpha+1-\theta)\right] \Gamma\left[\frac{1}{2}(\beta+\nu+\theta)\right]} R(x)=\phi(x)
$$

where

$$
R(x)=\Gamma(\mu+\nu+1) \frac{2}{\pi}^{\mu+\nu+1} x^{\beta+\nu} \int_{0}^{\infty} \frac{u^{\alpha+\mu}}{\left(x^{2}+u^{2}\right)^{\mu+\nu+1}} \phi(u) d u ;
$$

giving us an inversion of the generalized Potential transform.

The following special cases provide us with a procedure for inverting Laplace transform, cf. [3] and [6, p.232].

(ii) If $\alpha=\beta=\mu=\frac{1}{2}$ and $\nu=-\frac{1}{2}$, then Theorem 1 gives

THEOREM 2. Let $f$ and $\phi \in L^{2}(0, \infty)$. Define

$$
f(x)=\int_{0}^{\infty} e^{-x t} \phi(t) d t
$$

the bilateral Laplace transform of $\phi$. If

$$
R(x)=\frac{2}{\pi} \int_{0}^{\infty} \cos x t f(t) d t
$$

then

$$
\sin \frac{1}{2} \pi \theta[R(x)]=\phi(x), \text { a.a. } x>0
$$

Here the operator $\frac{1}{K(\theta)}$ is reduced to the operator $\sin \frac{1}{2} \pi \theta$. We can interpret this operator by considering the product expansion

$$
\sin \frac{1}{2} \pi z=\frac{1}{2} \pi z \lim _{n \rightarrow \infty} \prod_{k=1}^{n}\left(1-\frac{z^{2}}{4 k^{2}}\right) \text {. }
$$


Now if $S_{n}(\alpha)=\frac{1}{2} \pi \alpha \underset{k=1}{n}\left(1-\frac{\alpha^{2}}{4 k^{2}}\right)$, then, for example

$$
\sin \frac{1}{2} \pi \theta\left[x^{\alpha}\right]=\lim _{n \rightarrow \infty} S_{n}(-\alpha) x^{\alpha}=-\sin \frac{1}{2} \pi \alpha x^{\alpha}
$$

(iii) Put $\alpha=\mu=\frac{1}{2}$ and $\beta=\nu=\frac{1}{2}$ in Theorem 1, then we have

THEOREM 3. Let $f$ and $\phi \in L^{2}(0, \infty)$. Define

$$
f(x)=\int_{0}^{\infty} e^{-x t} \phi(t) d t,
$$

the Laplace transform of $\phi$. If

$$
R(x)=\frac{2}{\pi} \int_{0}^{\infty} \sin x t f(t) d t
$$

then

$$
\cos \frac{1}{2} \pi \theta[R(x)]=\phi(x), \quad \text { a.a. } x>0
$$

Here again the differential operator $\frac{1}{K(\theta)}$ of Theorem 1 is reduced to $\cos \frac{1}{2} \pi \theta$, which can be interpreted in a similar way as the operator $\sin \frac{1}{2} \pi \theta$. Also, for instance, $\cos \frac{1}{2} \pi \theta\left[x^{\alpha}\right]=\cos \frac{1}{2} \pi \alpha x^{\alpha}$.

(iv) Hamburger's formula.

EXAMPLE 2. Let $\phi(x)=\sin ^{2 n} x$. Then by Theorem 3,

$$
f(x)=\int_{0}^{\infty} \sin ^{2 n} t \quad e^{-x t} d t=\frac{(2 n) !}{x\left(x^{2}+2^{2}\right)\left(x^{2}+4^{2}\right) \cdots\left(x^{2}+(2 n)^{2}\right)},
$$

(Hamburger's formula).

Now

$$
\begin{aligned}
R(x) & =\frac{2(2 n) !}{\pi} \int_{0}^{\infty} \frac{\sin x t}{t\left(t^{2}+2^{2}\right)\left(t^{2}+4^{2}\right) \cdots\left(t^{2}+4 n^{2}\right)} d t \\
& =(-1)^{n} 2^{-2 n}\left[2 \sum_{k=0}^{n-1}(-1)^{k}\left(\begin{array}{c}
2 n \\
k
\end{array}\right) e^{2(k-n) x}+(-1)^{n}\left(\begin{array}{c}
2 n \\
n
\end{array}\right)\right],
\end{aligned}
$$

$[2, \mathrm{p} .414]$.

Thus, according to Theorem 3 


$$
\cos \frac{1}{2} \pi \theta[R(x)]=(-1)^{n} 2^{-2 n}\left[2 \sum_{k=0}^{n-1}(-1)^{k}\left(\begin{array}{c}
2 n \\
k
\end{array}\right) \cos \frac{1}{2} \pi \theta\left[e^{2(k-n) x}\right]+(-1)^{n}\left(\begin{array}{c}
2 n \\
n
\end{array}\right)\right]
$$

Now,

$$
\begin{aligned}
\cos \frac{1}{2} \pi \theta\left[e^{m x}\right] & =\cos \frac{1}{2} \pi \theta \sum_{k=0}^{\infty} \frac{(m x)^{k}}{k !} \\
& =\sum_{k=0}^{\infty} \frac{m^{k}}{k !} \cos \frac{1}{2} \pi \theta\left[x^{k}\right] \\
& =\sum_{k=0}^{\infty} \frac{m^{k}}{k !} \cos \frac{1}{2} \pi k x^{k}=\sum_{k=0}^{\infty} \frac{m^{2 k}(-1)^{k} n^{2 k}}{(2 k) !} \\
& =\cos m x .
\end{aligned}
$$

Hence

$$
\begin{aligned}
\cos \frac{1}{2} \pi \theta[R(x)] & =(-1)^{n} 2^{-2 n}\left[2 \sum_{k=0}^{n-1}(-1)^{k}\left(\begin{array}{c}
2 n \\
k
\end{array}\right) \cos [2(k-n) n]+(-1)^{n}\left(\begin{array}{c}
2 n \\
n
\end{array}\right)\right] \\
& =\sin ^{2 n} x,
\end{aligned}
$$

[2, p.25], as predicted, by the theorem, verifying the Hamburger formula.

\section{REFERENCES}

1. Erdelyi, A. et al. Tables of integral transforms, Vols. I and II, McGraw-Hill, New York, 1954.

2. Gradshteyn, I.S. and Ryzhik, I.M. Table of integral, series and products, (Translation) Academic Press, New York, 1965.

3. Haimo, D.T. and Cholewinski, F.M. Inversion of the reduced Poisson-Hankel transform, J. d'Analyse Math. 25 (1972).

4. NASIM, C. An inversion formula for Hankel transform, Pacific J. of Math. 57 (1) (1975).

5. Tichmarsh, E.C. Introduction to the theory of Fourier integrals, Second Edition, Clarendon Press, Oxford, England. 6. Widder, D.V. An introduction to transform theory, Academic Press, New York,
1971. 


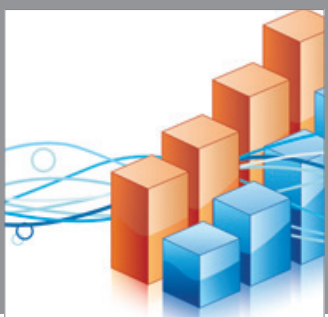

Advances in

Operations Research





\section{The Scientific} World Journal
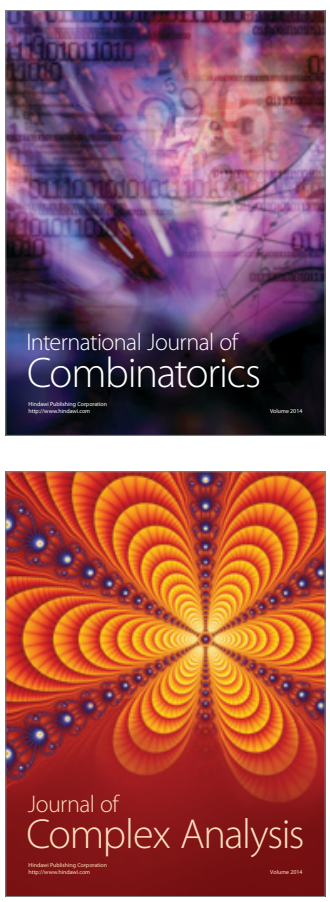

International Journal of

Mathematics and

Mathematical

Sciences
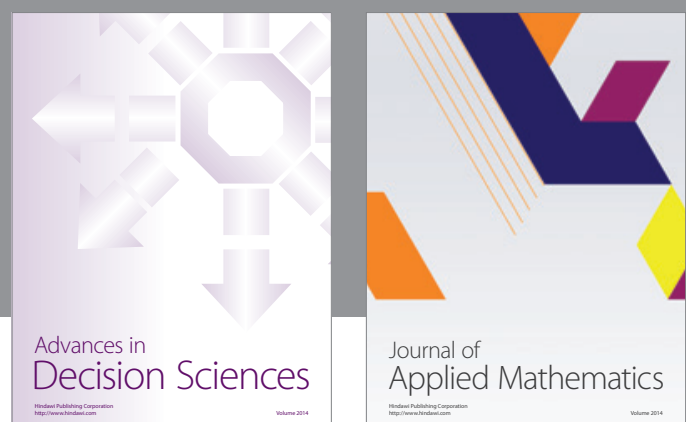

Journal of

Applied Mathematics
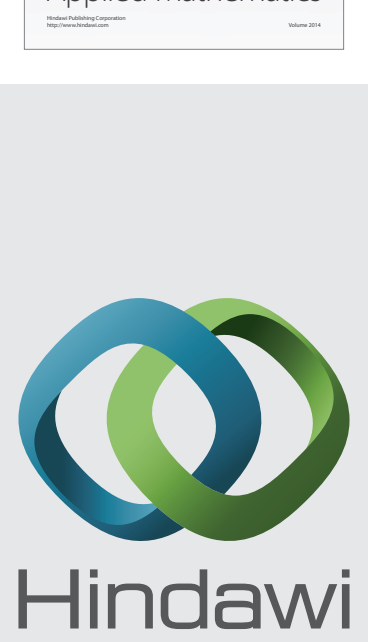

Submit your manuscripts at http://www.hindawi.com
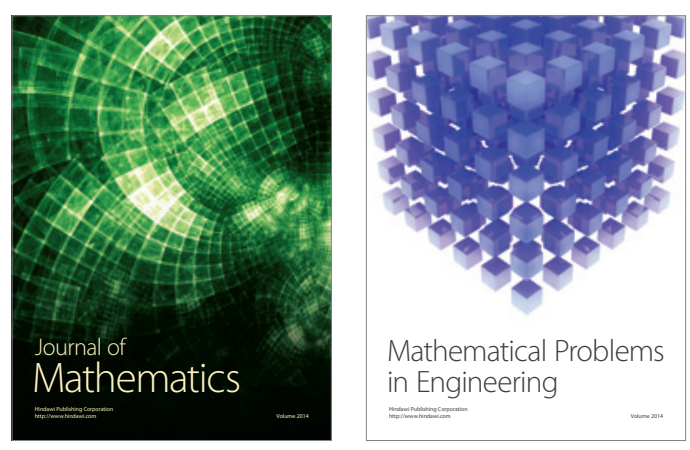

Mathematical Problems in Engineering


Journal of

Function Spaces
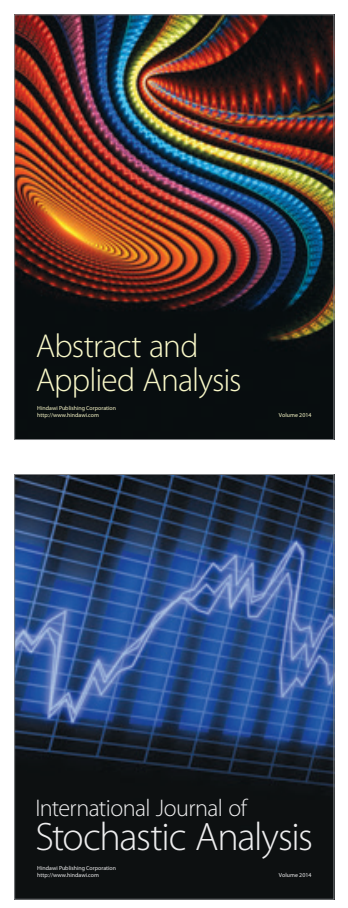

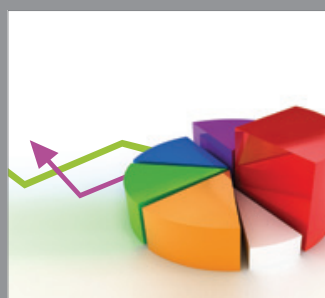

ournal of

Probability and Statistics

Promensencen
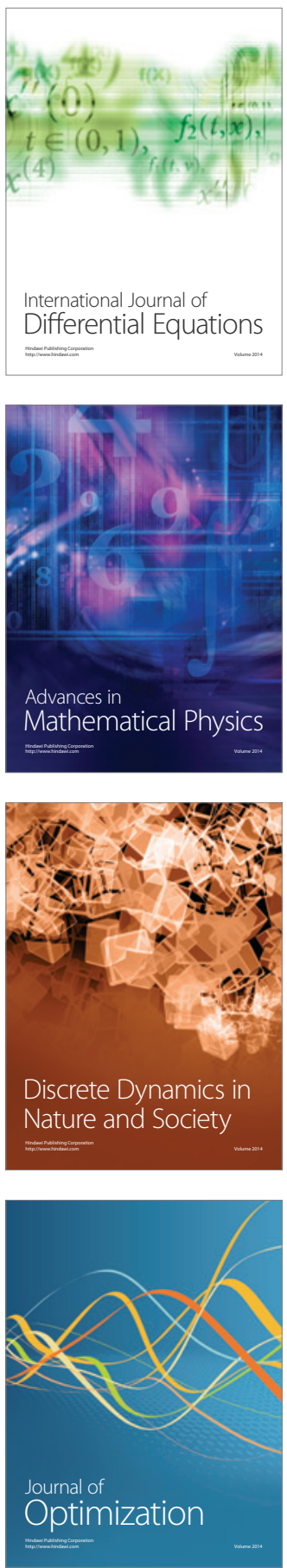